\title{
ON THE EVOLUTION OF ACCRETION DISKS IN GALACTIC CENTERS.
}

\author{
Wolfgang J. DUSCHL, \\ Institut für Theoretische Astrophysik, Universität Heidelberg, \\ Im Neuenheimer Feld 561, D-6900 Heidelberg 1, West Germany.
}

\begin{abstract}
.
I present the results of hydrodynamical model calculations for the evolution of accretion disks in an originally soft potential well. We follow the onset of selfgravity in the accretion disk and the formation of a central object, and find that for an ample set of possible parameter combinations of the "host galaxy" the evolution runs such that today, i.e. after an evolution time of the order of $10^{10} \mathrm{yrs}$, compact objects of about $10^{6} \mathrm{M} \odot$ have formed in the centers of galaxies. In the first Section, I concisely describe the basic ideas; Sect. 2 is devoted to the presentation of the results of hydrodynamical model calculations; and in the last Section, I shall discuss these results, interpreting them with the help of an analytical model that shows why the evolving central object is so independent of the conditions in the galaxy.
\end{abstract}

\section{BASIC IDEAS OF THE MODEL.}

I start with a galaxy with a soft potential in its central regions, i.e. $\lim _{r \rightarrow 0} \frac{d \Phi(r)}{d r}=0(r$ : radial coordinate in a spherical coordinate system; $\Phi(r)$ : gravitational potential); such a galaxy has no central object that dominates $\Phi$. Assuming that the galaxy also shows an overall rotation, $v_{\phi}(s)$, that is constant on cylinders of radius $s$ and directed in $\phi$-direction (cylindrical coordinate system $(s, z, \phi)$ ) a symmetry plane is defined in the galaxy.

Matter lost at a rate $\dot{\mu}$ from the stellar component of the galactic mass distribution may fall into this symmetry plane conserving its angular momentum. Thus a gas disk is built there. The mass distribution of this disk depends on the rotation law of the galaxy, the mass distribution of the stellar component, and the effective mass loss rate (effective means that not necessarily all matter lost by a star will fall into the disk, but only a fraction of it).

As we are running the disk (at least the inner parts of it) in a soft potential well, the usual radial viscous transport of matter and angular momentum will work only on time scales that are long compared to the Hubble time (Icke, 1979; Duschl, 1988a, 1988b). One can define an accretion time scale, $\tau_{a c c r}(s)$, that describes the time scale within which material moves from some radius $s$ in the disk to the center:

$$
\tau_{a c c r}(s)=\frac{1}{\alpha h(s) v_{c}(s)}\left|\frac{\frac{d\left(s^{2} \Omega(s)\right)}{d s}}{\frac{d \Omega(s)}{d s}}\right| ;
$$

$\alpha$ is a parameter describing the efficiency of viscosity: $\alpha=t_{s \phi} / P,\left(t_{s \phi}: s\right.$ - $\phi$-component of the stress tensor; $P$ : total pressure), $h$ the pressure scale hight (in $z$-direction) of the disk, $v_{c}$ the sound velocity, and $\Omega$ the equilibrium (Keplerian) angular frequency in the disk. $\alpha$ may or may not be constant, it is only the quotient as defined above. So it is not necessary to think of it in terms of turbulent 
viscosity (Shakura and Sunyaev, 1973), it could equally well be regarded as magnetic viscosity (Stella and Rosner, 1984; Coroniti, 1985) or as a viscosity induced by selfgravity (Paczyński, 1978; Lin and Pringle, 1987), for instance.

As $\tau_{\text {acer }}$ becomes extremely long in the central regions of the disk, there will be almost no radial flow. This means that matter that fell into the disk at some radius will remain there. Thus matter is piled up in the disk. Depending on the mass infall rate, after some time the disk will become selfgravitating. This will be the case as soon as the mass in the disk within some radius is no longer negligible compared to the mass of the background galaxy within the same radius. Now not only the galaxy determines the potential but also the mass of the disk plays a crucial role.

The whole process can be summarized as follows: Mass falls from the galaxy into the disk which it reaches at a smaller radius than the one it came from. There it piles up leading to a gas disk where larger and larger inner regions become selfgravitating. Within these regions a redistribution of matter towards the center becomes possible while the outer - not yet selfgravitating - zones stay inert with respect to accretion. This means that in this process not only the matter just falling into the selfgravitating parts of the disk participates in the accretion process but also the matter fallen into the disk when it still was dominated by the soft potential. The mass moving towards the center can there form a compact central object.

\section{NUMERICAL RESULTS.}

In the following I present the results of onedimensional implicit hydrodynamical model calculations of the evolution of accretion disks under the circumstances described in the previous Section. I assume a galaxy with a mass density distribution, $\rho(r)$, of

$$
\rho(r)=530 \mathrm{M} \odot / p c^{3} \cdot\left(1+\left(\frac{r}{100 p c}\right)^{2}\right)^{-\frac{3}{2}}
$$

Furthermore I assume for the rotation of the galaxy:

$$
v_{\phi}(s)= \begin{cases}2 \cdot 10^{7}(\mathrm{~s} / 1 \mathrm{kpc}) \mathrm{cm} / \mathrm{s}, & \text { if } s \leq 1 \mathrm{kpc} \\ 2 \cdot 10^{7} \mathrm{~cm} / \mathrm{s} & \text { if } s>1 \mathrm{kpc} .\end{cases}
$$

The effective mass loss rate, $\dot{\mu}^{*}$, is taken to be constant: $\dot{\mu}^{*}=7 \cdot 10^{-20} s^{-1}$.

Finally, it may happen that, within the selfgravitating accretion disk, stars form out of the disk's material. Here we assume the instantaneous recycling approximation; we shall show as an extreme example the results for the very short time scale of $\tau_{*}=3 \cdot 10^{15} \mathrm{~s}$, where $\tau_{*}$ is a time scale describing the evolution of the stellar phase. Stars are assumed to have that long an accretion time scale that accretion of these stars can be neglected.

As an example here I present results for a viscosity parameter of $\alpha=1$. I also assume that there is no heating of the disk from outside.

Fig. 1 gives the evolution of the central object for the two cases with no star formation at all, and with the very efficient one as defined above. Also other changes of parameters show how insensitive the evolution of the central object is to changes of the parameters of the environment (Duschl, 1988b). Taking the parameters of the galaxy as introduced above we find an original mass distribution of the galaxy as shown by the full line in Fig.2; after an evolution of $10^{10} \mathrm{yrs}$ the central region has changed quite drastically: we have now three domains: in the innermost the central object dominates (dotted line), further outwards the disk is the main contributor (dashed line) (this is mainly the selfgravitating region), and for even larger radii the background mass is practically unaltered by the disk. Comparison of these results with observed values (e.g. Genzel and Townes, 1987) show remarkable agreement. 
Figure 1: Comparison of the evolution of the mass of the central object for a model without star formation, and for a model with - highly efficient - star formation (see above).
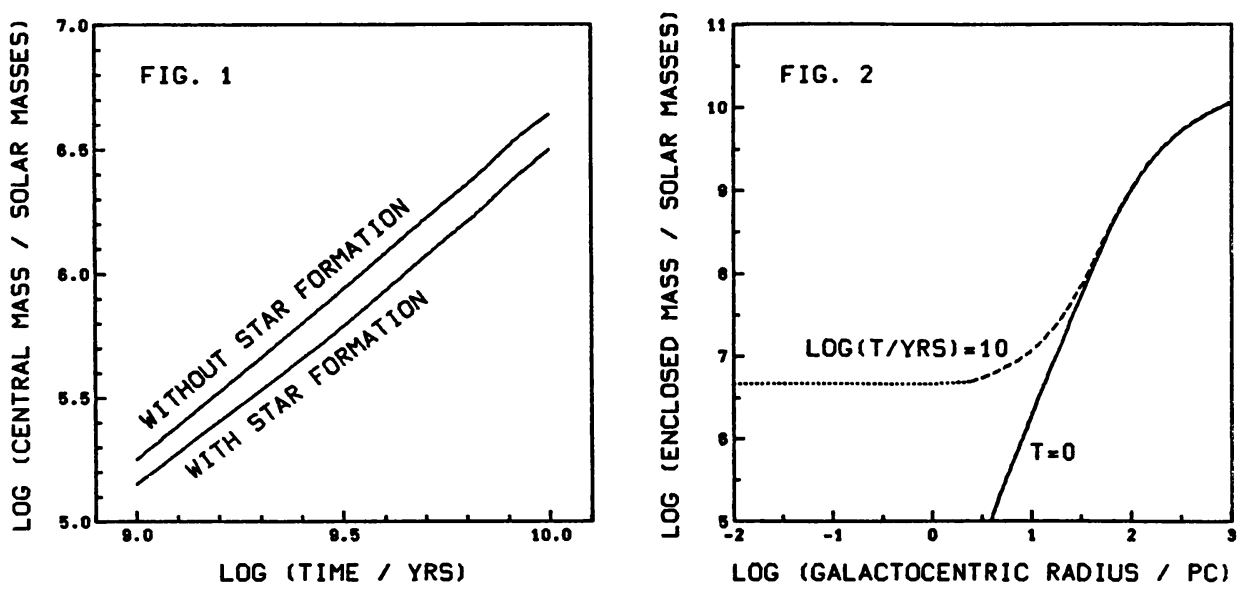

Figure 2: Mass distribution in the central $1 \mathrm{kpc}$ for the model discussed above (without star formation). The solid line marks the region where the - original - mass distribution in the galaxy dominates, the dashed one the region where the accretion disk is the main contributor to the enclosed mass, and the dotted line the region where the central object dominates the distribution.

\section{DISCUSSION AND AN ANALYTICAL MODEL.}

The numerical models show that a) for an ample set of parameters the resulting central objects have very similar masses, and that $b$ ) the central mass distribution is in agreement with what one observes in our galaxy, and also with the central masses other normal galaxies seem to show. In the following we shall develop an analytical model that allows deeper insight into which physical situations play a role and which do not.

At a certain radius, $s$, the mass of the galaxy within it, $M_{g a l}(s)$, the mass of the disk, $M_{d}(s)$, within it, and of the central object $M_{c}(s)$, determine the equilibrium angular velocity, $\Omega(s)$ :

$$
\Omega^{2}(s)=\frac{G M_{i n}(s)}{s^{3}}+\frac{G M_{g a l}(s)}{s^{3}},
$$

where $M_{\text {in }}(s)=M_{c}+M_{d}(s)$. Here we have neglected the fact that the mass of the disk is not distributed spherically symmetrically but is much more a very thin sheet; this introduces errors that are small enough not to influence the results of this analytical model. At the beginning of the evolution we have $M_{c}=M_{d}(s)=0$.

Accretion (i.e. redistribution of matter) is possible in a disk region where the total mass of disk and central object is comparable to the mass of the galaxy in the same region:

$$
M_{\text {gal }}\left(s_{a c c r}\right)=\xi M_{\text {in }}\left(s_{a c c r}\right),
$$

with $O(\xi) \nless 1$, and $O(\xi) \ngtr 1$. This defines the radius of the region within which accretion is possible, $s_{a c c r}$. In the following we regard only a disk averaged between $s=0$ and $s=s_{a c c r}$. We have

$$
M_{i n}(s)=\pi s_{a c c r}^{2} \Sigma_{a v}\left(s_{a c c r}\right) ; \quad \Sigma_{a v}\left(s_{a c c r}\right)=\Sigma_{i n}\left(s_{a c c r}\right) \cdot \sigma,
$$


$\Sigma_{a v}$ being the average surface density in this region parameterized in terms of the surface density at the outer edge. $\Sigma_{i n}$ is the surface density of the matter that already fell to the respective radius; as at $s_{a c c r}$ accretion just starts, this is the relevant surface density. As we are regarding the situation where we really have disk accretion and not almost a spherical accretion, and as the background galaxy has a monotonically distributed star phase, we can assume that $O(\sigma) \nless 1$, and $O(\sigma) \ngtr 1$.

We allow for a fraction $\gamma$ of the gas to become stars, and assume that stars only influence the potential but are not accreted. Then we have a gas component, $M_{d, g}$, of the disk material of

$$
M_{d, g}(s)=\gamma M_{d}(s),
$$

where $\gamma \leq 1$, and $O(\gamma) \nless 1$. For a selfgravitating disk one has

$$
h(s)=\frac{v_{c}^{2}(s)}{2 \pi G \Sigma(s)} .
$$

Now we assume that the gas mass within a radius $s$ can be accreted within the time scale $\tau_{a c c r}$ (cf. Eq.(1)), and get an average accretion rate, $\dot{M}$ :

$$
\dot{M}=\frac{M_{d, g}\left(s_{a c c r}\right)}{\tau_{a c c r}\left(s_{a c c r}\right)}
$$

and finally

$$
\dot{M}=\frac{\alpha v_{c}^{3}}{G} \cdot\left(1-\frac{M_{c}}{M_{i n}}\right) \cdot \phi, \quad \phi=\frac{\sigma \cdot(3 \sigma-1) \cdot \gamma}{4 \xi \sigma+\sigma+1}
$$

This result means that the accretion rate almost only depends on the viscosity - as long as $M_{c} \not M_{i n}$, and if $O(\phi) \nless 1$, and $O(\phi) \gg 1$. The dependence is twofold: directly via the parameter $\alpha$ that describes the viscosity's efficiency, and indirectly via the sound velocity, $v_{c}$, that might very well be also dominated by viscous heating. On the other side here we may also have a coupling to the environment in which the disk runs as this environment may heat the disk.

It is very crucial that we are dealing with a selfgravitating disk; were the disk not selfgravitating then either accretion would not set in at all, or - if at the end of the evolution selfgravity disappears the mass flow rate would change. The latter one will be the case only at much later stages than the ones discussed in this paper; even earlier the term $1-M_{c} / M_{i n}$ in Eq.(2) plays a role.

This analytical result is of the same type as what one knows from the spherically symmetric collapse of an isothermal sphere; in that case the accretion rate onto the central condensation also scales as $v_{c}^{3}$ (eg., Zinnecker and Tscharnuter, 1984).

\section{REFERENCES.}

Coroniti, F.V.: 1985, in: Unstable Current Systems and Plasma Instabilities in Astrophysics, Eds.:

Kundu, M.R., Holman, G.D., p.453

Duschl, W.J.: 1988a, Astron. Astrophys. 194,33

Duschl, W.J.: 1988b, Astron. Astrophys. 194,43

Genzel, R., Townes, C.H.: 1987, Ann.Rev.Astron.Astrophys. 25,377

Icke, V.: 1979, Astron. Astrophys. 78,21

Lin, D.N.C., Pringle, J.E.: 1987, Monthly Notices Royal Astron. Soc. 225,607

Paczyński, B.: 1978, Acta Astron. 28,91

Shakura, N.I., Sunyaev, R.A.: 1973: Astron. Astrophys. 24,337

Stella, L., Rosner, R.: 1984, Astrophys. J. 277,312

Zinnecker, H., Tscharnuter, W.M.: 1984, in: Workshop on Star Formation, Ed.: Wolstencroft, R.D., Edinburgh, Scotland, p.83 